\title{
INTEGERS WITH A MAXIMAL NUMBER OF FIBONACCI REPRESENTATIONS
}

\author{
Petra Kocábová ${ }^{1}$, Zuzana Masáková ${ }^{1}$ And \\ Edita Pelantová ${ }^{1}$
}

\begin{abstract}
We study the properties of the function $R(n)$ which determines the number of representations of an integer $n$ as a sum of distinct Fibonacci numbers $F_{k}$. We determine the maximum and mean values of $R(n)$ for $F_{k} \leq n<F_{k+1}$.
\end{abstract}

Mathematics Subject Classification. 11A67, 11B39.

\section{INTRODUCTION}

Let $\left(F_{k}\right)_{k \geq 0}$ be the Fibonacci sequence defined by

$$
F_{0}=F_{1}=1, \quad F_{k+1}=F_{k}+F_{k-1} \quad \text { for } k \geq 1 .
$$

Every positive integer $n$ can be written as a sum of distinct Fibonacci numbers, i.e. in the form

$$
n=F_{m_{r}}+F_{m_{r-1}}+\cdots+F_{m_{1}}, \quad \text { where } m_{r}>m_{r-1}>\cdots>m_{1} \geq 1 .
$$

The expression (1) is called a representation of the number $n$ in the Fibonacci number system. The index of the maximal Fibonacci number that appears in the representation of $n$ is called the length of the representation. Every Fibonacci representation can be written in the form of a finite word $w=w_{m_{r}} w_{m_{r}-1} \ldots w_{1}$ in the alphabet $\{0,1\}$, where $w_{i}=1$ for $i=m_{1}, \ldots, m_{r}$, and $w_{i}=0$ otherwise. For example the number $n=32$ can be represented as

$$
32=21+5+3+2+1=F_{7}+F_{4}+F_{3}+F_{2}+F_{1}
$$

Keywords and phrases. Fibonacci numbers, Zeckendorf representation.

1 Department of Mathematics, FNSPE, Czech Technical University, Trojanova 13, 12000 Praha 2, Czech Republic; petra.kocabova@centrum.cz, masakova@km1.fjfi.cvut.cz, pelantova@km1.fjfi.cvut.cz

(C) EDP Sciences 2005 
and this representation corresponds to the word 1001111. Due to the recurrence relation for Fibonacci numbers, different representations of the number $n$ can be obtained by substituting the string 011 by 100 and vice versa. All representations of 32 correspond to words

\section{0, 1010011, 1001111, 111111.}

The number of different Fibonacci representations of $n$ will be denoted by $R(n)$. Let us enumerate the first twenty values of the sequence $(R(n))_{n \geq 1}$,

$$
(R(n))_{n \geq 1}=1,1,2,1,2,2,1,3,2,2,3,1,3,3,2,4,2,3,3,1, \ldots
$$

For a given positive integer $n$ we can find $k$ such that

$$
F_{k} \leq n<F_{k+1}
$$

It is obvious that every representation of $n$ has length $\leq k$. On the other hand, since

$$
F_{1}+F_{2}+\cdots+F_{k-2}<F_{k} \leq n,
$$

the lengths of every representation of $n$ is at least $k-1$. Thus representations of the number $n$ can be divided into long (having length $k$ ) and short (of length $k-1$ ). Let us denote by $R_{1}(n)$ the number of long representations of $n$, and by $R_{0}(n)$ the number of short representations of $n$. Clearly

$$
R(n)=R_{1}(n)+R_{0}(n) .
$$

If we prefix the short Fibonacci representations of $n$ with the prefix 0 , they have the same length as the long representations of $n$. The lexicographically greatest among all such representations of the number $n$ is called the Zeckendorf representation of $n$ and the corresponding word in the alphabet $\{0,1\}$ is denoted by $\langle n\rangle$. The distinguishing characteristic of this representation is that there are no adjacent 1 's. For example, we have $\langle 32\rangle=1010100$.

The Zeckendorf representation of a number $n$ is a word of the form

$$
\langle n\rangle=10^{r_{1}} 10^{r_{2}} \ldots 10^{r_{l}}, \quad \text { where } r_{1}, \ldots, r_{l-1} \geq 1 \text {, and } r_{l} \geq 0 \text {. }
$$

The sum $r_{1}+r_{2}+\cdots+r_{l}+l$ determines the length of the Zeckendorf representation of $n$. Since the relation between the number $n$ and the word (3) is one-to-one, we define for the simplicity of notation

$$
\begin{aligned}
\varrho\left(r_{1}, \ldots, r_{l}\right) & :=R(n) \\
\varrho_{1}\left(r_{1}, \ldots, r_{l}\right) & :=R_{1}(n) \\
\varrho_{0}\left(r_{1}, \ldots, r_{l}\right) & :=R_{0}(n)
\end{aligned}
$$

where $\langle n\rangle=10^{r_{1}} 10^{r_{2}} \ldots 10^{r_{l}}$. 
It can be seen easily that $R(n)=1$ if and only if $n=F_{k}-1$ for some $k \geq 2$. The values of $R(n)$ for $n=F_{k} \pm j, j \leq 8$ are given in [3]. The segment of the sequence $R(n)$ between two consecutive occurrences of 1 is a palindrome [3,4], i.e.

$$
R\left(F_{k}-1+i\right)=R\left(F_{k+1}-1-i\right), \quad \text { for } i=1,2, \ldots, F_{k-1}-1 .
$$

The aim of this paper is to find the maximal and the mean values of the function $R(n)$ for $F_{k}-1<n<F_{k+1}-1$, which corresponds to the numbers $n$ whose Zeckendorf representation has a fixed length $k$. We determine the numbers

$$
\begin{aligned}
\operatorname{Max}(k) & :=\max \left\{R(n) \mid n \in \mathbb{N}, F_{k} \leq n<F_{k+1}\right\} \\
& =\max \left\{\varrho\left(r_{1}, \ldots, r_{l}\right) \mid l \in \mathbb{N}, r_{1}, \ldots, r_{l-1} \geq 1, r_{l} \geq 0, l+\sum_{i=1}^{l} r_{i}=k\right\} .
\end{aligned}
$$

In addition, we classify the arguments of the maxima.

Let us determine several initial values of the sequence $\operatorname{Max}(k)$. It suffices to divide the sequence $(R(n))_{n \geq 1}$ to blocks of length $F_{0}, F_{1}, F_{2}, \ldots$ along the occurrence of consecutive 1's and to find maximal values in these blocks, see (2). We have

$$
\begin{array}{ll}
\operatorname{Max}(1)=\max \{R(n) \mid 1 \leq n<2\} & =R(1)=1, \\
\operatorname{Max}(2)=\max \{R(n) \mid 2 \leq n<3\} & =R(2)=1, \\
\operatorname{Max}(3)=\max \{R(n) \mid 3 \leq n<5\} & =R(3)=2, \\
\operatorname{Max}(4)=\max \{R(n) \mid 5 \leq n<8\} & =R(5)=R(6)=2, \\
\operatorname{Max}(5)=\max \{R(n) \mid 8 \leq n<13\} & =R(8)=R(11)=3, \\
\operatorname{Max}(6)=\max \{R(n) \mid 13 \leq n<21\} & =R(16)=4 .
\end{array}
$$

\section{Properties of the Functions $\varrho, \varrho_{0}, \varrho_{1}$}

Berstel [1] gives an explicit formula for computing the values of functions $\varrho, \varrho_{1}$, $\varrho_{0}$ defined in (4). Denote the matrix

$$
M(r):=\left(\begin{array}{cc}
\left\lceil\frac{r}{2}\right\rceil & \left\lfloor\frac{r}{2}\right\rfloor \\
1 & 1
\end{array}\right) .
$$

Theorem 2.1 (Berstel). Let $r_{1}, \ldots, r_{l} \in \mathbb{Z}, r_{1}, \ldots, r_{l-1} \geq 1, r_{l} \geq 0$. Then

$$
\left(\begin{array}{l}
\varrho_{0}\left(r_{1}, \ldots, r_{l}\right) \\
\varrho_{1}\left(r_{1}, \ldots, r_{l}\right)
\end{array}\right)=M\left(r_{1}\right) M\left(r_{2}\right) \ldots M\left(r_{l}\right)\left(\begin{array}{l}
0 \\
1
\end{array}\right) .
$$


Since $\varrho\left(r_{1}, \ldots, r_{l}\right)=\varrho_{0}\left(r_{1}, \ldots, r_{l}\right)+\varrho_{1}\left(r_{1}, \ldots, r_{l}\right)$, we have explicit formulas for the functions $\varrho, \varrho_{0}, \varrho_{1}$ in the following form

$$
\begin{gathered}
\varrho\left(r_{1}, \ldots, r_{l}\right)=\left(\begin{array}{ll}
1 & 1
\end{array}\right) M\left(r_{1}\right) M\left(r_{2}\right) \ldots M\left(r_{l}\right)\left(\begin{array}{l}
0 \\
1
\end{array}\right), \\
\varrho_{0}\left(r_{1}, \ldots, r_{l}\right)=\left(\begin{array}{ll}
1 & 0
\end{array}\right) M\left(r_{1}\right) M\left(r_{2}\right) \ldots M\left(r_{l}\right)\left(\begin{array}{l}
0 \\
1
\end{array}\right), \\
\varrho_{1}\left(r_{1}, \ldots, r_{l}\right)=\left(\begin{array}{ll}
0 & 1
\end{array}\right) M\left(r_{1}\right) M\left(r_{2}\right) \ldots M\left(r_{l}\right)\left(\begin{array}{l}
0 \\
1
\end{array}\right) .
\end{gathered}
$$

Let us now derive some recurrence relations for $\varrho\left(r_{1}, \ldots, r_{l}\right)$ that will be needed for determining the maximal values. If $l=1$ we get directly from (6) that

$$
\varrho(r)=\left\lfloor\frac{r}{2}\right\rfloor+1
$$

Lemma 2.2. Let $l \in \mathbb{N}$, and let $r_{1}, r_{2}, \ldots, r_{l} \in \mathbb{Z}, r_{1}, r_{2}, \ldots, r_{l-1} \geq 1, r_{l} \geq 0$. If $r_{l}$ is odd, then $\varrho\left(r_{1}, \ldots, r_{l}\right)=\varrho\left(r_{1}, \ldots, r_{l}-1\right)$.

Proof. It follows from (6) since for $r_{l}$ odd we have $M\left(r_{l}\right)\left(\begin{array}{l}0 \\ 1\end{array}\right)=M\left(r_{l}-1\right)\left(\begin{array}{l}0 \\ 1\end{array}\right)$.

Lemma 2.3. Let $l \in \mathbb{N}, l \geq 2$ and let $r_{1}, r_{2}, \ldots, r_{l} \in \mathbb{Z}, r_{1}, r_{2}, \ldots, r_{l-1} \geq 1$, $r_{l} \geq 0$. If $r_{i}$ is even for some $1 \leq i \leq l-1$, then

$$
\varrho\left(r_{1}, \ldots, r_{l}\right)=\varrho\left(r_{1}, \ldots, r_{i}\right) \varrho\left(r_{i+1}, \ldots, r_{l}\right) .
$$

Proof. For $r_{i}$ even we have $M\left(r_{i}\right)=M\left(r_{i}\right)\left(\begin{array}{l}0 \\ 1\end{array}\right)\left(\begin{array}{ll}1 & 1\end{array}\right)$. Substituting into (6) we obtain the lemma.

Lemma 2.4. Let $l \in \mathbb{N}, l \geq 2$, and let $r_{1}, r_{2}, \ldots, r_{l} \in \mathbb{Z}, r_{1}, r_{2}, \ldots, r_{l-1} \geq 1$, $r_{l} \geq 0$. We have

$$
\begin{array}{ll}
\varrho\left(r_{1}, r_{2}, \ldots, r_{l}\right)=\frac{r_{1}+1}{2} \varrho\left(r_{2}, \ldots, r_{l}\right)+\varrho_{0}\left(r_{2}, \ldots, r_{l}\right), & \text { if } r_{1} \text { is odd, } \\
\varrho\left(r_{1}, r_{2}, \ldots, r_{l}\right)=\left(\frac{r_{1}}{2}+1\right) \varrho\left(r_{2}, \ldots, r_{l}\right), & \text { if } r_{1} \text { is even } .
\end{array}
$$

Proof. First suppose $r_{1}$ is odd. Since

$$
\left(\begin{array}{ll}
1 & 1
\end{array}\right) M\left(r_{1}\right)=\frac{r_{1}+1}{2}\left(\begin{array}{ll}
1 & 1
\end{array}\right)+\left(\begin{array}{ll}
1 & 0
\end{array}\right)
$$

substituting into (6) gives the desired result. The statement for $r_{1}$ even is a consequence of Lemma 2.3 and the relation (7).

Lemma 2.5. Let $l \in \mathbb{N}, l \geq 3$, and let $r_{1}, r_{2}, \ldots, r_{l} \in \mathbb{Z}, r_{1}, r_{2}, \ldots, r_{l-1} \geq 1$, $r_{l} \geq 0$. If for some $i, 2 \leq i \leq l-1$, the coefficient $r_{i}$ is odd, then

$\varrho\left(r_{1}, \ldots, r_{l}\right)=\varrho\left(r_{1}, \ldots, r_{i}-1\right) \varrho\left(r_{i+1}, \ldots, r_{l}\right)+\varrho\left(r_{1}, \ldots, r_{i-1}+1\right) \varrho_{0}\left(r_{i+1}, \ldots, r_{l}\right)$. 
Proof. Again, it suffices to verify the matrix equality

$$
M\left(r_{i-1}\right) M\left(r_{i}\right)=M\left(r_{i-1}\right) M\left(r_{i}-1\right)\left(\begin{array}{l}
0 \\
1
\end{array}\right)\left(\begin{array}{ll}
1 & 1
\end{array}\right)+M\left(r_{i-1}+1\right)\left(\begin{array}{l}
0 \\
1
\end{array}\right)\left(\begin{array}{ll}
1 & 0
\end{array}\right)
$$

for $r_{i}$ odd and to use (6).

The following lemma is a direct consequence of the definition of functions $\varrho, \varrho_{0}$ and can be found in [4] as Lemma 1.

Lemma 2.6 (Edson, Zamboni). Let $l \in \mathbb{N}, l \geq 2$, and let $r_{1}, r_{2}, \ldots, r_{l} \in \mathbb{Z}$, $r_{1}, r_{2}, \ldots, r_{l-1} \geq 1, r_{l} \geq 0$. Then

(i) $\varrho_{0}\left(r_{1}, r_{2}, \ldots, r_{l}\right)=\varrho\left(r_{1}-2, r_{2}, \ldots, r_{l}\right)$, for $r_{1} \geq 3$;

(ii) $\varrho_{0}\left(2, r_{2}, \ldots, r_{l}\right)=\varrho\left(r_{2}, \ldots, r_{l}\right)$;

(iii) $\varrho_{0}\left(1, r_{2}, \ldots, r_{l}\right)=\varrho_{0}\left(r_{2}, \ldots, r_{l}\right)$;

(iv) $\varrho\left(r_{1}, \ldots, r_{l-1}, 1,1, \ldots, 1\right)=\varrho\left(r_{1}, \ldots, r_{l-1}\right)$.

Clearly, $\varrho\left(r_{1}, \ldots, r_{l}\right) \geq 1$. However, the number of short Fibonacci representations $\varrho_{0}\left(r_{1}, \ldots, r_{l}\right)$ can be equal to 0 . Using the rules given in Lemma 2.6 we easily deduce that

$$
\varrho_{0}\left(r_{1}, \ldots, r_{l}\right)=0 \quad \Longleftrightarrow \quad r_{1}=r_{2}=\cdots=r_{l-1}=1 \text { and } r_{l} \in\{0,1\} .
$$

\section{LOWER BOUND ON $\operatorname{Max}(k)$}

In order to find the lower estimates of $\operatorname{Max}(k)$, let us determine the values $\varrho\left(r_{1}, \ldots, r_{l}\right)$ on some chosen $l$-tuples $\left(r_{1}, \ldots, r_{l}\right)$.

\section{Lemma 3.1.}

1) $\varrho(\underbrace{3,3, \ldots, 3}_{k-1 \text { times }}, 4)=\varrho(1, \underbrace{3, \ldots, 3}_{k-1 \text { times }}, 2)=F_{2 k+1} \quad$ for $k \geq 1$.

2) $\varrho(\underbrace{3,3, \ldots, 3}_{k \text { times }}, 2)=\varrho(1, \underbrace{3, \ldots, 3}_{k-1 \text { times }}, 4)=F_{2 k+2} \quad$ for $k \geq 1$.

Proof. Let us first show by induction that for the $s$-th power of the matrix $M(3)=\left(\begin{array}{ll}2 & 1 \\ 1 & 1\end{array}\right)$ we have

$$
(M(3))^{s}=\left(\begin{array}{cc}
F_{2 s} & F_{2 s-1} \\
F_{2 s-1} & F_{2 s-2}
\end{array}\right), \quad \text { for } s \in \mathbb{N} .
$$

For $s=1$ the statement is trivial. For $s \geq 2$ we use the induction hypothesis

$$
(M(3))^{s}=(M(3))^{s-1}\left(\begin{array}{ll}
2 & 1 \\
1 & 1
\end{array}\right)=\left(\begin{array}{ll}
F_{2 s-2} & F_{2 s-3} \\
F_{2 s-3} & F_{2 s-4}
\end{array}\right)\left(\begin{array}{ll}
2 & 1 \\
1 & 1
\end{array}\right)=\left(\begin{array}{cc}
F_{2 s} & F_{2 s-1} \\
F_{2 s-1} & F_{2 s-2}
\end{array}\right) .
$$


Note that $(9)$ is valid also for $s=0$ if we define $F_{-1}, F_{-2}$ in such a way that the recurrence relation is still valid, $\left(F_{-1}=0, F_{-2}=1\right)$. It is now easy to use (6) to find

$$
\begin{aligned}
\varrho(\underbrace{3,3, \ldots, 3}_{k-1 \text { times }}, 4) & =\left(\begin{array}{ll}
1 & 1
\end{array}\right)\left(\begin{array}{ll}
F_{2 k-2} & F_{2 k-3} \\
F_{2 k-3} & F_{2 k-4}
\end{array}\right)\left(\begin{array}{ll}
2 & 2 \\
1 & 1
\end{array}\right)\left(\begin{array}{l}
0 \\
1
\end{array}\right) \\
& =\left(\begin{array}{ll}
F_{2 k-1} & F_{2 k-2}
\end{array}\right)\left(\begin{array}{l}
2 \\
1
\end{array}\right)=F_{2 k+1}
\end{aligned}
$$

and

$$
\begin{aligned}
\varrho(1, \underbrace{3, \ldots, 3}_{k-1 \text { times }}, 2) & =\left(\begin{array}{ll}
1 & 1
\end{array}\right)\left(\begin{array}{ll}
1 & 0 \\
1 & 1
\end{array}\right)\left(\begin{array}{ll}
F_{2 k-2} & F_{2 k-3} \\
F_{2 k-3} & F_{2 k-4}
\end{array}\right)\left(\begin{array}{ll}
1 & 1 \\
1 & 1
\end{array}\right)\left(\begin{array}{l}
0 \\
1
\end{array}\right) \\
& =\left(\begin{array}{ll}
F_{2 k} & F_{2 k-1}
\end{array}\right)\left(\begin{array}{l}
1 \\
1
\end{array}\right)=F_{2 k+1} .
\end{aligned}
$$

The relations (2) can be proved similarly.

As a corollary, we have a lower estimate on the maxima for numbers with Zeckendorf representation of odd length.

Corollary 3.2. $\operatorname{Max}(2 k+1) \geq F_{k+1}$ for $k \geq 1$.

From the definition of the function $\varrho$ it follows that

$$
\varrho\left(2, r_{1}, \ldots, r_{l}\right) \geq 2 \varrho\left(r_{1}, \ldots, r_{l}\right)
$$

and for $r_{l}>0$ also

$$
\varrho\left(r_{1}, \ldots, r_{l}, 2\right) \geq 2 \varrho\left(r_{1}, \ldots, r_{l}\right) .
$$

Therefore we have the following lower estimate on the maxima for numbers with Zeckendorf representation of even length.

Corollary 3.3. $\operatorname{Max}(2 k+2) \geq 2 \operatorname{Max}(2 k-1) \geq 2 F_{k}$ for $k \geq 2$.

Our aim is to show that the inequalities in Corollaries 3.2 and 3.3 are in fact equalities.

\section{MAXima of the FUNCTION $R(n)$}

Let us now determine the maximum of the function $R(n)=\varrho\left(r_{1}, r_{2}, \ldots, r_{l}\right)$, where $F_{k} \leq n<F_{k+1}$ and $\langle n\rangle=10^{r_{1}} 10^{r_{2}} \ldots 10^{r_{l}}$. The $l$-tuple $r_{1}, \ldots, r_{l} \in \mathbb{Z}$ must satisfy $r_{1}, r_{2}, \ldots, r_{l-1} \geq 1, r_{l} \geq 0$ and $\sum_{i=1}^{l} r_{i}+l=k$. We shall not repeat these assumptions.

Let us show that $\operatorname{Max}(k)$ is not reached on integers $n$ whose Zeckendorf representation has only one 1 . More precisely, we have the following proposition.

Proposition 4.1. Let $\operatorname{Max}(k)=\varrho\left(r_{1}, r_{2}, \ldots, r_{l}\right)$. Then $l \geq 2$ or $k \leq 5$. 
Proof. Suppose by contradiction that $k \geq 6$ and $l=1$. Then using (7), we have $\operatorname{Max}(k)=\varrho(k-1)=\left\lfloor\frac{k-1}{2}\right\rfloor+1$. For $k$ even we have by Corollary 3.3

$$
2 F_{\frac{k-2}{2}} \leq \operatorname{Max}(k)=\left\lfloor\frac{k-1}{2}\right\rfloor+1=\frac{k}{2}
$$

which is in contradiction with $2 F_{i-1}>i$ for all $i \geq 3$. For $k$ odd we have by Corollary 3.2

$$
F_{\frac{k+1}{2}} \leq \operatorname{Max}(k)=\left\lfloor\frac{k-1}{2}\right\rfloor+1=\frac{k+1}{2},
$$

which contradicts the fact that $F_{i}>i$ for all $i \geq 4$.

In the following several propositions we show that the maximum is reached on $l$-tuples of a certain specific form. The proofs are done by contradiction. Assuming that the maximal $l$-tuple does not satisfy the desired properties, we find another $l$-tuple on which the function $\varrho$ has strictly greater value.

Proposition 4.2. Let $\operatorname{Max}(k)=\varrho\left(r_{1}, r_{2}, \ldots, r_{l}\right)$ for $k \geq 6$. Then $r_{l}$ is even.

Proof. Since the above Proposition 4.1 implies that $l \geq 2$, it suffices to prove that for $r_{l}$ odd we have

$$
\varrho\left(r_{1}, r_{2}, \ldots, r_{l-1}, r_{l}\right)<\varrho\left(r_{1}+1, r_{2}, \ldots, r_{l-1}, r_{l}-1\right) .
$$

We divide the demonstration of (10) into two cases.

a) Let $r_{1}$ be even. Using Lemmas 2.2 and 2.4 we have

$$
\begin{aligned}
& \varrho\left(r_{1}, r_{2}, \ldots, r_{l-1}, r_{l}\right)=\varrho\left(r_{1}, r_{2}, \ldots, r_{l-1}, r_{l}-1\right) \\
& =\left(\frac{r_{1}}{2}+1\right) \varrho\left(r_{2}, \ldots, r_{l-1}, r_{l}-1\right), \\
& \varrho\left(r_{1}+1, r_{2}, \ldots, r_{l-1}, r_{l}-1\right) \\
& \quad=\frac{r_{1}+2}{2} \varrho\left(r_{2}, \ldots, r_{l-1}, r_{l}-1\right)+\varrho_{0}\left(r_{2}, \ldots, r_{l-1}, r_{l}-1\right) .
\end{aligned}
$$

In order to obtain $(10)$ we need to show that $\varrho_{0}\left(r_{2}, \ldots, r_{l-1}, r_{l}-1\right)>0$. Using (8), $\varrho_{0}\left(r_{2}, \ldots, r_{l-1}, r_{l}-1\right)=0$ with $r_{l}$ odd implies $r_{2}=r_{3}=$ $\cdots=r_{l}=1$. However, in this case the property (iv) of Lemma 2.6 and Proposition 4.1 give

$$
\varrho\left(r_{1}, r_{2}, \ldots, r_{l-1}, r_{l}\right)=\varrho\left(r_{1}, 1,1, \ldots, 1\right)=\varrho\left(r_{1}\right)<\varrho(k-1)<\operatorname{Max}(k),
$$

which contradicts the assumption of the proposition. Thus we necessarily have $\varrho_{0}\left(r_{2}, \ldots, r_{l-1}, r_{l}-1\right)>0$ and $(10)$ is valid. 
b) Let $r_{1}$ be odd. Again we use Lemmas 2.2 and 2.4 to obtain

$$
\begin{aligned}
& \varrho\left(r_{1}, r_{2}, \ldots, r_{l-1}, r_{l}\right)=\frac{r_{1}+1}{2} \varrho\left(r_{2}, \ldots, r_{l-1}, r_{l}\right)+\varrho_{0}\left(r_{2}, \ldots, r_{l-1}, r_{l}\right) \\
& \varrho\left(r_{1}+1, r_{2}, \ldots, r_{l-1}, r_{l}-1\right)=\varrho\left(r_{1}+1, \ldots, r_{l}\right)=\left(\frac{r_{1}+1}{2}+1\right) \varrho\left(r_{2}, \ldots, r_{l}\right) .
\end{aligned}
$$

The validity of $(10)$ is obvious, since $\varrho\left(r_{2}, \ldots, r_{l}\right)>\varrho_{0}\left(r_{2}, \ldots, r_{l}\right)$.

In order to find the arguments of the maxima of the function $\varrho$, we use the matrix formula (6). First we introduce a partial ordering on non-negative matrices. Lemma 4.4 then shows that replacing a matrix in (6) by a "bigger" one increases the value of the function $\varrho$.

Definition 4.3. Let $\mathbb{X}=\left(\begin{array}{ll}a & b \\ c & d\end{array}\right)$ and $\tilde{\mathbb{X}}=\left(\begin{array}{ll}\tilde{a} & \tilde{b} \\ \tilde{c} & \tilde{d}\end{array}\right)$ be integer matrices with nonnegative components. We say that $\mathbb{X}$ majores $\tilde{\mathbb{X}}$ (written $\mathbb{X} \succ \tilde{\mathbb{X}}$ ) if

$$
a \geq \tilde{a}, \quad b \geq \tilde{b}, \quad a+c \geq \tilde{a}+\tilde{c} \quad \text { and } \quad b+d>\tilde{b}+\tilde{d}
$$

Lemma 4.4. Let $\alpha=\left(\begin{array}{ll}1 & 1\end{array}\right) \mathbb{A} \mathbb{X} \mathbb{B}\left(\begin{array}{l}0 \\ 1\end{array}\right)$ and $\tilde{\alpha}=\left(\begin{array}{ll}1 & 1\end{array}\right) \mathbb{A} \tilde{\mathbb{X}} \mathbb{B}\left(\begin{array}{l}0 \\ 1\end{array}\right)$, where

$$
\begin{array}{lll}
\mathbb{A}=\mathbb{I}_{2} & \text { or } & \mathbb{A}=M\left(r_{1}\right) \ldots M\left(r_{s}\right) . \\
\mathbb{B}=\mathbb{I}_{2} & \text { or } & \mathbb{B}=M\left(p_{1}\right) \ldots M\left(p_{t}\right),
\end{array}
$$

and $\mathbb{X}, \tilde{\mathbb{X}}$ are non-negative integer matrices. If $\mathbb{X} \succ \tilde{\mathbb{X}}$, then $\alpha>\tilde{\alpha}$.

Proof. Denote $\left(\begin{array}{ll}x & y\end{array}\right)=\left(\begin{array}{ll}1 & 1\end{array}\right) \mathbb{A}$ and $\left(\begin{array}{l}z \\ u\end{array}\right)=\mathbb{B}\left(\begin{array}{l}0 \\ 1\end{array}\right)$. It is easy to see that $x \geq y \geq 1$ and that $z \geq 0, u \geq 1$. Let $\mathbb{X}=\left(\begin{array}{ll}a & b \\ c & d\end{array}\right)$ and $\tilde{\mathbb{X}}=\left(\begin{array}{ll}a & \tilde{b} \\ \tilde{c} & \tilde{d}\end{array}\right)$ satisfy (11). Then

$$
\begin{aligned}
& \alpha-\tilde{\alpha}=\left(\begin{array}{ll}
x & y
\end{array}\right)\left(\begin{array}{ll}
a & b \\
c & d
\end{array}\right)\left(\begin{array}{l}
z \\
u
\end{array}\right)-\left(\begin{array}{ll}
x & y
\end{array}\right)\left(\begin{array}{ll}
\tilde{a} & \tilde{b} \\
\tilde{c} & \tilde{d}
\end{array}\right)\left(\begin{array}{l}
z \\
u
\end{array}\right) \\
& =((a-\tilde{a}) x+(c-\tilde{c}) y,(b-\tilde{b}) x+(d-\tilde{d}) y)\left(\begin{array}{l}
z \\
u
\end{array}\right) \\
& \geq((a+c-\tilde{a}-\tilde{c}) y,(b+d-\tilde{b}-\tilde{d}) y)\left(\begin{array}{l}
z \\
u
\end{array}\right) \geq\left(\begin{array}{ll}
0 & 1
\end{array}\right)\left(\begin{array}{l}
0 \\
1
\end{array}\right)=1 \text {. }
\end{aligned}
$$

Proposition 4.5. Let $\varrho\left(r_{1}, r_{2}, \ldots, r_{l}\right)=\operatorname{Max}(k)$. Then $r_{i} \leq 5$ for all $i=$ $1,2, \ldots, l$.

Proof. Let $\langle n\rangle=10^{r_{1}} 10^{r_{2}} \cdots 10^{r_{l}}$, and assume that there exists an index $i$ such that $r_{i} \geq 6$. Denote by $m$ the number with Zeckendorf representation $\langle m\rangle=$ $10^{r_{1}} \cdots 10^{r_{i-1}} 10^{r_{i}-3} 10^{2} 10^{r_{i+1}} \cdots 10^{r_{l}}$. Zeckendorf representations $\langle n\rangle$ and $\langle m\rangle$ 
have the same length. Since

$$
\begin{aligned}
M\left(r_{i}\right) & =\left(\begin{array}{cc}
\left\lceil\frac{r_{i}}{2}\right\rceil & \left\lfloor\frac{r_{i}}{2}\right\rfloor \\
1 & 1
\end{array}\right) \prec\left(\begin{array}{cc}
\left\lceil\frac{r_{i}-3}{2}\right\rceil & \left\lfloor\frac{r_{i}-3}{2}\right\rfloor \\
1
\end{array}\right)\left(\begin{array}{ll}
1 & 1 \\
1 & 1
\end{array}\right) \\
& =\left(\begin{array}{cc}
r_{i}-3 & r_{i}-3 \\
2 & 2
\end{array}\right)=M\left(r_{i}-3\right) M(2),
\end{aligned}
$$

we have according to Lemma 4.4

$$
R(n)=\varrho\left(r_{1}, r_{2}, \ldots, r_{l}\right)<\varrho\left(r_{1}, \ldots, r_{i-1}, r_{i}-3,2, r_{i+1}, \ldots, r_{l}\right)=R(m),
$$

which contradicts the assumption of the proposition.

Proposition 4.6. Let $\varrho\left(r_{1}, r_{2}, \ldots, r_{l-1}, r_{l}\right)=\operatorname{Max}(k)$, where $k \geq 6$ and the $r_{i}$ are odd for $i=1,2, \ldots, l-1$. Then $r_{1} \in\{1,3\}, r_{2}, \ldots, r_{l-1}=3$, and $r_{l} \in\{2,4\}$.

Proof. As a consequence of Proposition 4.2, the final coefficient $r_{l}$ is even, and due to Proposition 4.5 it can take only values $\{0,2,4\}$. Assumption of the present proposition with Proposition 4.5 implies that $r_{1}, r_{2}, \ldots, r_{l-1} \in\{1,3,5\}$. First let us show by contradiction that 5 does not occur. Suppose the opposite, i.e. that there exists an index $1 \leq i \leq l-1$ such that $r_{i}=5$. Let $i$ be the maximal index with this property. Let $s$ be the minimal non-negative integer, such that $r_{i+s} \neq 3$. Then $r_{i+s}=1$ or $i+s=l$ and $r_{i+s} \in\{0,2,4\}$.

1) Let $r_{i+s}=1$. We verify that

$$
\tilde{\mathbb{X}}=M(5)(M(3))^{s-1} M(1) \prec(M(3))^{s+1}=\mathbb{X} .
$$

According to (9), we obtain

$$
\tilde{\mathbb{X}}=\left(\begin{array}{ll}
3 & 2 \\
1 & 1
\end{array}\right)\left(\begin{array}{ll}
F_{2 s-2} & F_{2 s-3} \\
F_{2 s-3} & F_{2 s-4}
\end{array}\right)\left(\begin{array}{ll}
1 & 0 \\
1 & 1
\end{array}\right)=\left(\begin{array}{cc}
F_{2 s+2} & F_{2 s} \\
F_{2 s} & F_{2 s-2}
\end{array}\right), \quad \mathbb{X}=\left(\begin{array}{cc}
F_{2 s+2} & F_{2 s+1} \\
F_{2 s+1} & F_{2 s}
\end{array}\right) .
$$

Obviously $\tilde{\mathbb{X}} \prec \mathbb{X}$ and using Lemma 4.4 we obtain

$$
\begin{aligned}
\operatorname{Max}(k) & =\varrho(r_{1}, \ldots, r_{i-1}, 5, \underbrace{3, \ldots, 3}_{s-1 \text { times }}, 1, r_{i+s+1}, \ldots, r_{l}) \\
& <\varrho(r_{1}, \ldots, r_{i-1}, \underbrace{3, \ldots, 3}_{s+1 \text { times }}, r_{i+s+1}, \ldots, r_{l})
\end{aligned}
$$

which is a contradiction.

2) Let $r_{i+s}=2$. Similarly as in (1) we use matrices and Lemma 4.4 to obtain the contradiction

$$
\operatorname{Max}(k)=\varrho(r_{1}, \ldots, r_{i-1}, 5, \underbrace{3, \ldots, 3}_{s-1 \text { times }}, 2)<\varrho(r_{1}, \ldots, r_{i-1}, \underbrace{3, \ldots, 3}_{s \text { times }}, 4) .
$$


3) Let $r_{i+s}=4$. Similarly as in (1) we use matrices and Lemma 4.4 to obtain the contradiction

$$
\operatorname{Max}(k)=\varrho(r_{1}, \ldots, r_{i-1}, 5, \underbrace{3, \ldots, 3}_{s-1 \text { times }}, 4)<\varrho(r_{1}, \ldots, r_{i-1}, \underbrace{3, \ldots, 3}_{s+1 \text { times }}, 2) .
$$

4) Let $r_{i+s}=0$. Similarly as in (1) we use matrices and Lemma 4.4 to obtain the contradiction

$$
\operatorname{Max}(k)=\varrho(r_{1}, \ldots, r_{i-1}, 5, \underbrace{3, \ldots, 3}_{s-1 \text { times }}, 0)<\varrho(r_{1}, \ldots, r_{i-1}, \underbrace{3, \ldots, 3}_{s \text { times }}, 2) .
$$

Thus we have shown that $r_{1}, \ldots, r_{l-1} \leq 3$, i.e. all take values in $\{1,3\}$.

Let us now prove by contradiction that at most one of the coefficients $r_{1}, \ldots, r_{l-1}$ is equal to 1 . Assume that there exist indices $i, i+s, 1 \leq i<i+s \leq l-1$ such that $r_{i}=r_{i+s}=1$ and $r_{i+1}=r_{i+2}=\cdots=r_{i+s-1}=3$. Denote

$$
\begin{aligned}
& \tilde{\mathbb{X}}=M(1)(M(3))^{s-1} M(1)=\left(\begin{array}{cc}
F_{2 s-1} & F_{2 s-3} \\
F_{2 s} & F_{2 s-2}
\end{array}\right), \\
& \mathbb{X}=(M(3))^{s}=\left(\begin{array}{cc}
F_{2 s} & F_{2 s-1} \\
F_{2 s-1} & F_{2 s-2}
\end{array}\right) .
\end{aligned}
$$

Since $\tilde{\mathbb{X}} \prec \mathbb{X}$, we derive that

$$
\begin{aligned}
\operatorname{Max}(k) & =\varrho(r_{1}, \ldots, r_{i-1}, 1, \underbrace{3, \ldots, 3}_{s-1 \text { times }}, 1, r_{i+s+1, \ldots, r_{l}}) \\
& <\varrho(r_{1}, \ldots, r_{i-1}, \underbrace{3, \ldots, 3}_{s \text { times }}, r_{i+s+1}, \ldots, r_{l}),
\end{aligned}
$$

which contradicts the maximality of $\varrho\left(r_{1}, \ldots, r_{l}\right)$. Thus at most one of the coefficients $r_{1}, \ldots, r_{l-1}$ is equal to 1 and the others are equal to 3 .

If $l=2$, the proposition is proved. For $l \geq 3$ we show by contradiction that $r_{2}=\cdots=r_{l-1}=3$. Suppose that $r_{i}=1$ for some $2 \leq i \leq l-1$. Since

$$
\begin{aligned}
& \left(\begin{array}{ll}
1 & 1
\end{array}\right)(M(3))^{i-1} M(1)=\left(F_{2 i} F_{2 i-2}\right), \\
& \left(\begin{array}{lll}
1 & 1
\end{array}\right) M(1)(M(3))^{i-1}=\left(F_{2 i} F_{2 i-1}\right),
\end{aligned}
$$

it follows that

$$
\operatorname{Max}(k)=\varrho(\underbrace{3, \ldots, 3}_{i-1 \text { times }}, 1, r_{i+1}, \ldots, r_{l})<\varrho(1, \underbrace{3, \ldots, 3}_{i-1 \text { times }}, r_{i+1}, \ldots, r_{l}),
$$

which is a contradiction.

It remains to show that $r_{l} \neq 0$. But if $r_{l}=0$, then $r_{l-1}=3$. Relation $M(3) M(0) \prec M(4)$ implies a contradiction. 
We are now in position to state the theorem about the maximal values of $R(n)$.

\section{Theorem 4.7.}

$$
\begin{aligned}
& \max \left\{R(n) \mid F_{2 k+1} \leq n<F_{2 k+2}\right\}=\operatorname{Max}(2 k+1)=F_{k+1} \quad \text { for } k \geq 0, \\
& \max \left\{R(n) \mid F_{2 k+2} \leq n<F_{2 k+3}\right\}=\operatorname{Max}(2 k+2)=2 F_{k} \quad \text { for } k \geq 1 .
\end{aligned}
$$

Proof. In the proof we shall make use of the following inequalities for Fibonacci numbers, which are not difficult to demonstrate.

$$
F_{x+1} F_{y+1} \leq 2 F_{x+y} \quad \text { for } \quad x, y \geq 0,
$$

where the equality holds only if $x=1$ or $y=1$.

$$
2 F_{x} F_{y} \leq F_{x+y+1} \quad \text { for } x, y \geq 1,
$$

where the equality holds only if $x=y=2$.

Since the lower bounds on the maxima of the function $R(n)$ are known from Corollaries 3.2 and 3.3 , it suffices to prove inequalities

$$
\operatorname{Max}(2 k+1) \leq F_{k+1} \quad \text { and } \quad \operatorname{Max}(2 k+2) \leq 2 F_{k} .
$$

Let us show it by induction on $k$. For initial values of $k$ the validity of the theorem follows from (5). Now assume that

$$
\operatorname{Max}(2 j+1) \leq F_{j+1} \quad \text { and } \quad \operatorname{Max}(2 j+2) \leq 2 F_{j}, \quad \text { for } j<k .
$$

With this induction hypothesis we want to show (14).

- Let us first show that $\operatorname{Max}(2 k+2) \leq 2 F_{k}$.

Let $r_{1}, r_{2}, \ldots, r_{l}$ be an $l$-tuple such that $\varrho\left(r_{1}, r_{2}, \ldots, r_{l}\right)=\operatorname{Max}(2 k+2)$ where $k \geq 2$. Proposition 4.2 implies that $r_{l}$ is even. Since $r_{1}+r_{2}+\cdots+r_{l}+l=2 k+2$, there must exist an $i<l$ such that $r_{i}$ is even. Let $i$ be the maximal $i<l$ with this property. The number $r_{i+1}+\cdots+r_{l}+(l-i)$ is odd, say $2 m+1$. Then $r_{1}+\cdots+r_{i}+i=2 k+2-(2 m+1)$. Lemma 2.3 , the induction hypothesis and inequality (12) implies

$$
\begin{aligned}
\operatorname{Max}(2 k+2) & =\varrho\left(r_{1}, \ldots, r_{l}\right)=\varrho\left(r_{1}, \ldots, r_{i}\right) \varrho\left(r_{i+1}, \ldots, r_{l}\right) \\
& \leq \operatorname{Max}(2 k-2 m+1) \operatorname{Max}(2 m+1)=F_{k-m+1} F_{m+1} \leq 2 F_{k} .
\end{aligned}
$$

- Now let us show the inequality $\operatorname{Max}(2 k+1) \leq F_{k+1}$.

Let $r_{1}, r_{2}, \ldots, r_{l}$ be an $l$-tuple such that $\varrho\left(r_{1}, r_{2}, \ldots, r_{l}\right)=\operatorname{Max}(2 k+1)$ where $k \geq 2$. Suppose that besides $r_{l}$ there exist another $i<l$ such that $r_{i}$ is even and let $i$ be the maximal index $i<l$ with this property. Let us denote $r_{i+1}+\cdots+r_{l}+(l-i)=$ 
$2 m+1$. Then $r_{1}+\cdots+r_{2}+i=2 k+1-(2 m+1)=2 k-2 m$. Lemma 2.3 , the induction hypothesis and inequality (13) implies

$$
\begin{aligned}
\operatorname{Max}(2 k+1) & =\varrho\left(r_{1}, \ldots, r_{l}\right)=\varrho\left(r_{1}, \ldots, r_{i}\right) \varrho\left(r_{i+1}, \ldots, r_{l}\right) \\
& \leq \operatorname{Max}(2 k-2 m) \operatorname{Max}(2 m+1)=2 F_{k-m-1} F_{m+1} \leq F_{k+1}
\end{aligned}
$$

It remains to consider the case that the $l$-tuple $r_{1}, r_{2}, \ldots, r_{l}$ which satisfies $\varrho\left(r_{1}, r_{2}, \ldots, r_{l}\right)=\operatorname{Max}(2 k+1)$ contains all $r_{i}$ odd for $1 \leq i \leq l-1$. According to Proposition 4.6 the maximal $l$-tuple is of the form $(1,3, \ldots, 3,4),(3, \ldots, 3,4)$, $(1,3, \ldots, 3,2)$, or $(3, \ldots, 3,2)$. Note that for fixed length of the Zeckendorf representation only two of these are possible, namely $(1,3, \ldots, 3,2)$, or $(3, \ldots, 3,4)$ for length $1 \bmod 4$, and $(1,3, \ldots, 3,4),(3, \ldots, 3,2)$ for length $3 \bmod 4$. The values of the function $\varrho$ for these $l$-tuples was determined in Lemma 3.1. Therefore the statement of the theorem is proved.

\section{Argument of $\operatorname{Max}(k)$}

In this section we determine the integers on which the maximum of the function $R(n)$ is reached for a fixed length $\sum_{i=1}^{l} r_{i}+l$ of the Zeckendorf representation $\langle n\rangle=10^{r_{1}} \cdots 10^{r_{l}}$. The proof of Theorem 4.7 allows us to determine the $l$-tuples $r_{1}, \ldots, r_{l}$ representing such integers $n$.

Suppose first that the Zeckendorf representation of $n$ has odd length. In this case the proof of Theorem 4.7 indicates that unless equality holds in (16), all the coefficients $r_{1}, \ldots, r_{l-1}$ are odd and therefore the $l$-tuples $r_{1}, \ldots, r_{l-1}, r_{l}$ are of very specific form (as a consequence of Prop. 4.6).

Equality in (16) provides an exceptional $l$-tuple. In order to make (16) true, the relation (13) necessitates that $k=4$ (hence $m=1$ ) and

$$
\varrho\left(r_{1}, \ldots, r_{i}\right)=\operatorname{Max}(6) \quad \text { and } \quad \varrho\left(r_{i+1}, \ldots, r_{l}\right)=\operatorname{Max}(3) \text {. }
$$

Since according to the table (5) we have $\operatorname{Max}(6)=R(16)=\varrho(2,2)$ and $\operatorname{Max}(3)=$ $R(3)=\varrho(2)$ necessarily $l=3$ and $r_{1}=r_{2}=r_{3}=2$.

\section{Corollary 5.1.}

(i) $\operatorname{Max}(4 k+3)$ is reached precisely on two arguments for $k \geq 1$ and on one argument for $k=0$. We have $\operatorname{Max}(3)=\varrho(2)$, and for $k \geq 1$

$$
\operatorname{Max}(4 k+3)=\varrho(1, \underbrace{3, \ldots, 3}_{k-1 \text { times }}, 4)=\varrho(\underbrace{3, \ldots, 3}_{k \text { times }}, 2) .
$$

(ii) $\operatorname{Max}(4 k+1)$ is reached precisely on two arguments for $k \geq 3$ or $k=1$, on three arguments for $k=2$, and on one argument for $k=0$. We have $\operatorname{Max}(1)=\varrho(0), \operatorname{Max}(9)=\varrho(1,3,2)=\varrho(3,4)=\varrho(2,2,2)$, and for $k=1$ 
and $k \geq 3$

$$
\operatorname{Max}(4 k+1)=\varrho(1, \underbrace{3, \ldots, 3}_{k-1 \text { times }}, 2)=\varrho(\underbrace{3, \ldots, 3}_{k-1 \text { times }}, 4) .
$$

As for integers with even length of their Zeckendorf representation, proof of Theorem 4.7 requires that an $l$-tuple $r_{1}, \ldots, r_{l}$ on which the maximum of $\varrho$ is reached must satisfy equality in (15). Relation (12) for Fibonacci numbers implies that $m-k=1$ or $m=1$. This can be true only if $i=1$ or $i=l-1$ respectively. Equality in (15) further requires that either $r_{1}=2, r_{2}, \ldots, r_{l}$ are odd and $\varrho\left(r_{2}, \ldots, r_{l}\right)$ is maximal, or $r_{l}=2$ and $\varrho\left(r_{2}, \ldots, r_{l}\right)$ is maximal, respectively.

Corollary 5.2. Let $k \geq 3$ and let $r_{1}, \ldots, r_{l}$ satisfy $\sum_{i=1}^{l} r_{i}+l=2 k$. Then $\varrho\left(r_{1}, \ldots, r_{l}\right)=\operatorname{Max}(2 k)$ if and only if

$$
\begin{aligned}
r_{1}=2 \quad \text { and } \quad \varrho\left(r_{2}, \ldots, r_{l}\right) & =\operatorname{Max}(2 k-3) \\
r_{l}=2 & \text { and } \quad \varrho\left(r_{1}, \ldots, r_{l-1}\right)=\operatorname{Max}(2 k-3) .
\end{aligned}
$$

Recall that the elements of the sequence $(R(n))_{n \in \mathbb{N}}$ can be grouped into palindromes $R\left(F_{k}\right), \ldots, R\left(F_{k+1}-2\right)$ separated by values $R\left(F_{k+1}-1\right)=1$. Corollaries 5.1 and 5.2 show that up to the exceptional initial cases, the maximal value in each palindrome occurs twice (for $k$ odd) and four times (for $k$ even). The description of arguments of the maxima of $R(n)$ in the palindrome, i.e. for $n$ with fixed length of Zeckendorf representation, is given in Theorem 5.3. We need to introduce the following notation,

$$
\begin{aligned}
i_{2 k+1} & = \begin{cases}F_{k+1} F_{k-3}+1 & \text { for } k \text { even, } \\
F_{k} F_{k-2}+1 & \text { for } k \text { odd },\end{cases} \\
i_{2 k} & = \begin{cases}F_{k+2} F_{k-5}+F_{3}+1 & \text { for } k \text { even, } \\
F_{k+1} F_{k-4}+F_{3}+1 & \text { for } k \text { odd },\end{cases} \\
j_{2 k} & = \begin{cases}F_{k+1} F_{k-3}+1 & \text { for } k \text { even, } \\
F_{k} F_{k-2}+1 & \text { for } k \text { odd } .\end{cases}
\end{aligned}
$$

Theorem 5.3.

(i) $\operatorname{Max}(2 k+1)$ for $k \geq 1, k \neq 4$ is reached precisely on the integers

$$
F_{2 k+1}-1+i_{2 k+1}, \quad F_{2 k+2}-1-i_{2 k+1} .
$$

For $k=4, \operatorname{Max}(2 k+1)=\operatorname{Max}(9)$ is reached precisely on three integers, namely

$$
F_{9}-1+i_{9}=63, \quad F_{10}-1-i_{9}=79, \quad \text { and their average } 71 .
$$


(ii) $\operatorname{Max}(2 k)$ for $k \geq 3, k \neq 6$, is reached precisely on the integers

$$
\begin{array}{ll}
F_{2 k}-1+i_{2 k}, & F_{2 k+1}-1-i_{2 k}, \\
F_{2 k}-1+j_{2 k}, & F_{2 k+1}-1-j_{2 k} .
\end{array}
$$

For $k=6, \operatorname{Max}(2 k)=\operatorname{Max}(12)$ is reached precisely on five integers, namely

$$
\begin{gathered}
F_{12}-1+i_{12}=270, \quad F_{13}-1-i_{12}=338, \\
F_{12}-1+j_{12}=296, \quad F_{13}-1-j_{12}=312, \\
\text { and their avarage } 304 .
\end{gathered}
$$

Proof. Corollaries 5.1 and 5.2 show that up to the exceptional initial cases, the maximal value in the palindrome $R\left(F_{k}\right), \ldots, R\left(F_{k+1}-2\right)$ occurs twice for $k$ odd and four times for $k$ even. From the symmetry of the palindrome, for $k$ odd there is an integer $i_{k} \in\left\{1,2, \ldots, F_{k-1}-1\right\}$ such that

$$
R\left(F_{k}-1+i_{k}\right)=R\left(F_{k+1}-1-i_{k}\right)=\operatorname{Max}(k) .
$$

Without loss of generality $i_{k}$ is in our considerations the smaller of the two integers satisfying it. Similarly, for $k$ even we have $i_{k}, j_{k} \in\left\{1,2, \ldots, F_{k-1}-1\right\}$ such that

$R\left(F_{k}-1+i_{k}\right)=R\left(F_{k+1}-1-i_{k}\right)=R\left(F_{k}-1+j_{k}\right)=R\left(F_{k+1}-1-j_{k}\right)=\operatorname{Max}(k)$.

We consider $i_{k}<j_{k}$ to be the two smallest of the four integers satisfying it.

We derive the compact form of $i_{k}$ and $j_{k}$ from arguments of maxima given in Corollaries 5.1 and 5.2. For that we use the relation

$$
F_{i}+F_{i+4}+F_{i+8}+\cdots+F_{i+4(k-1)}=F_{2 k+i-2} F_{2 k-1}, \quad \text { for } i, k \geq 1,
$$

which can be shown using $F_{k}=\frac{1}{\sqrt{5}}\left(\tau^{k+1}-{\tau^{\prime}}^{k+1}\right)$, where $\tau=\frac{1}{2}(1+\sqrt{5})$ is the golden ratio and $\tau^{\prime}=\frac{1}{2}(1-\sqrt{5})$ its algebraic conjugate.

It is interesting to study the position of the maximal values in the palindrome $R\left(F_{k}-1\right), R\left(F_{k}\right), \ldots, R\left(F_{k+1}-1\right)$, i.e. the position of integers $i_{k},\left(i_{k}\right.$ and $\left.j_{k}\right)$ in the set $1,2, \ldots, F_{k-1}$. This is described by Proposition 5.4 and illustrated in Figure 1.

Proposition 5.4. Let $k \geq 1$. Then

$$
\begin{array}{cc}
\lim _{k \rightarrow \infty} \frac{i_{2 k+1}}{F_{2 k}}=\lim _{k \rightarrow \infty} \frac{i_{2 k}}{F_{2 k-1}}=\frac{1}{\tau+2}, & \left|i_{2 k+1}-\frac{F_{2 k}}{\tau+2}\right|<\frac{1}{2}, \\
\lim _{k \rightarrow \infty} \frac{j_{2 k}}{F_{2 k-1}}=\frac{\tau}{\tau+2}, & \left|j_{2 k}-\frac{\tau F_{2 k-1}}{\tau+2}\right|<\frac{1}{2} .
\end{array}
$$




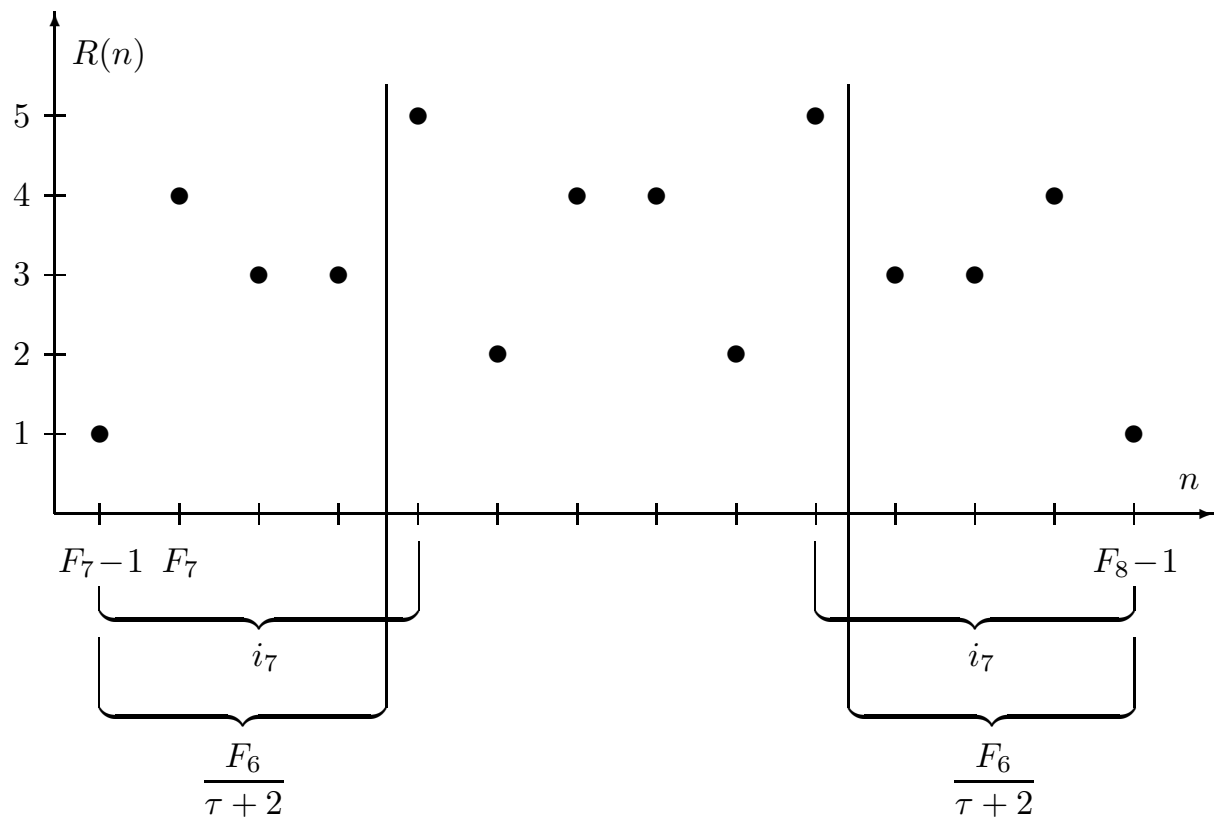

Figure 1. Illustration of the function $R(n)$ for $n \in[20,33]$. The values $R\left(F_{k}-1\right), R\left(F_{k}\right), \ldots, R\left(F_{k+1}-1\right)$ for $k=7$ form a palindrome. Since $k \equiv 3 \bmod 4$, the maximal value $\operatorname{Max}(7)$ appears twice and these local maxima are at the integers nearest to the asymptotical position, which is marked by the vertical lines.

The proposition shows that the numbers $i_{2 k+1}$ and $j_{2 k}$ are the closest integers to the asymptotic position of the maximal value. Let us mention that it is slightly more complicated in case of $i_{2 k}$.

Remark. Bicknell-Johnson defines in [2] a sequence $(A(q))_{q \in \mathbb{N}}$ which determines the smallest positive integer with $q$ Fibonacci representations and shows that

$$
A\left(F_{k}\right)=F_{k}^{2}-1 \quad \text { and } \quad A\left(2 F_{k}\right)=F_{k+3} F_{k}-1+(-1)^{K} .
$$

Since $F_{2 k+1}-1+i_{2 k+1}=F_{k+1}^{2}-1$ and $F_{2 k}-1+i_{2 k}=F_{k+2} F_{k-1}-1+(-1)^{k-1}$, the result of [2] is a consequence of Theorems 4.7 and 5.3.

\section{Mean value of $R(n)$}

Berstel in his article [1] states an open question about the mean value of the function $R(n)$. In this section we answer his question. In particular, we determine the mean value of $R(n)$ for integers with fixed length $k$ of their Zeckendorf 
representation, i.e. the value

$$
\frac{1}{F_{k-1}} \sum_{n=F_{k}}^{F_{k+1}-1} R(n)
$$

Proposition 6.1. Let $k \geq 1$. Then

$$
\sum_{n=F_{k}}^{F_{k+1}-1} R(n)=\frac{1}{3}\left(2^{k}-(-1)^{k}\right)
$$

Proof. Consider the word $w=w_{l} w_{l-1} \ldots w_{1}$ in the alphabet $\{0,1\}$ where $w_{l}=1$. The word $w$ is a representation of the number $n=\sum_{i=1}^{l} w_{i} F_{i}$. Also $w$ is a long representation of $n$, if $\sum_{i=1}^{l} w_{i} F_{i}<F_{l+1}$, and $w$ is a short representation of $n$, if $\sum_{i=1}^{l} w_{i} F_{i} \geq F_{l+1}$. It can be easily shown that the latter occurs if and only if the word $w$ has the prefix $1010 \cdots 1011$. More precisely,

$$
\sum_{i=1}^{l} w_{i} F_{i} \geq F_{l+1}
$$

if and only if

$$
w_{l} w_{l-1} \cdots w_{1}=(10)^{i} 11 w_{l-2 i-2} \cdots w_{1}, \quad \text { for some } i \geq 0, i \leq\left\lfloor\frac{l-2}{2}\right\rfloor
$$

Therefore the number of words $w_{l} \cdots w_{1}$ with $w_{l}=1$ that represent an integer $n \geq F_{l+1}$ is equal to the number of distinct suffixes $w_{l-2 i-2} \cdots w_{1}$, i.e.

$$
\sum_{i=0}^{\left\lfloor\frac{l-2}{2}\right\rfloor} 2^{l-2 i-2}=\left\lfloor\frac{2^{l}-1}{3}\right\rfloor
$$

Consequently, the number of words $w_{l} \cdots w_{1}$ with $w_{l}=1$ which represent an integer $n<F_{l+1}$ is equal to

$$
2^{l-1}-\left\lfloor\frac{2^{l}-1}{3}\right\rfloor
$$


Since the sets of Fibonacci representations of distinct integers $n$ are disjoint, we obtain

$$
\begin{aligned}
\sum_{n=F_{k}}^{F_{k+1}-1} R_{0}(n) & =\#\left\{w_{k-1} \cdots w_{1} \in\{0,1\}^{*} \mid w_{k-1}=1, \sum_{i=1}^{k-1} w_{i} F_{i} \geq F_{k}\right\} \\
& =\left\lfloor\frac{2^{k-1}-1}{3}\right\rfloor, \\
\sum_{n=F_{k}}^{F_{k+1}-1} R_{1}(n) & =\#\left\{w_{k} \cdots w_{1} \in\{0,1\}^{*} \mid w_{k}=1, \sum_{i=1}^{k} w_{i} F_{i}<F_{k+1}\right\} \\
& =2^{k-1}-\left\lfloor\frac{2^{k}-1}{3}\right\rfloor .
\end{aligned}
$$

Together we obtain

$$
\sum_{n=F_{k}}^{F_{k+1}-1} R(n)=2^{k-1}-\left\lfloor\frac{2^{k}-1}{3}\right\rfloor+\left\lfloor\frac{2^{k-1}-1}{3}\right\rfloor=\frac{1}{3}\left(2^{k}-(-1)^{k}\right) .
$$

Since $F_{k}=\frac{1}{\sqrt{5}}\left(\tau^{k+1}-\tau^{\prime k+1}\right)$, the mean value of the function $R(n)$ for $F_{k} \leq n<$ $F_{k+1}$ is equal to

$$
\frac{\frac{1}{3}\left(2^{k}-(-1)^{k}\right)}{\frac{1}{\sqrt{5}}\left(\tau^{k}-\tau^{\prime k}\right)} \sim \frac{\sqrt{5}}{3}\left(\frac{2}{\tau}\right)^{k}
$$

Acknowledgements. The results were derived using a computer program implemented by F. Maňák to whom the authors express their gratitude.

\section{REFERENCES}

[1] J. Berstel, An exercise on Fibonacci representations. RAIRO-Inf. Theor. Appl. 35 (2001) 491-498.

[2] M. Bicknell-Johnson, The smallest positive integer having $F_{k}$ representations as sums of distinct Fibonacci numbers, in Applications of Fibonacci numbers. Vol. 8, Kluwer Acad. Publ., Dordrecht (1999) 47-52.

[3] M. Bicknell-Johnson and D.C. Fielder, The number of representations of $N$ using distinct Fibonacci numbers, counted by recursive formulas. Fibonacci Quart. 37 (1999) 47-60.

[4] M. Edson and L. Zamboni, On representations of positive integers in the Fibonacci base. Preprint University of North Texas (2003).

Communicated by J. Berstel.

Received February 17, 2004. Accepted June 8, 2004. 